\title{
Study on Model and Simulation of the Tilt Rotor Aircraft in Transition Mode
}

\author{
Laohu Yuan, Wenhao Zhang, Xin Wen \\ School of Shenyang Aerospace University, Shenyang 110136, China. \\ hszdzwh@163.com
}

Keywords: tilt rotor aircraft; transition mode; dynamic model.

\begin{abstract}
In recent years, tilt rotor aircraft has been greatly developed because of its excellent performance. However, because of the complicacy in transition mode study on this aircraft is needed. In this paper a six degrees of freedom dynamic model of the tilt rotor aircraft was established, and PID controller was chosen when the aircraft in transition mode. Meanwhile, a defined S-function was used to solve the nonlinearity of the dynamic equations in real time and a simulation model is built in Matlab/Simulink. The results of simulation show the system is stable and provide a reference for analysis of tilt rotor aircraft dynamic and design of its flight control system.
\end{abstract}

\section{Introduction}

Tilt rotor aircraft, combined the vertical lift ability of helicopters and the speed of fixed-wing aircrafts, has been a great development in recent years [1]. And it also has a bright future in military and civilian application [2]. Researchers at home and abroad have made a great number of researches on the aircraft dynamic model [3-6]. However, most of them build the model using linearization techniques which make the result of simulation inaccurate because of the ignoring of high order element.

In this paper, tilt rotor aircraft is a main research object, then its force and moment in the transition mode are analyzed. A six degree of freedom dynamic model of the aircraft is established by the Newton-Euler method. And a PID controller is used to make its attitude tend to stable quickly. In order to solve nonlinearity of the model, a defined S-function is used to express the state equations when the simulation model is established [7].

\section{Dynamic model}

Firstly, the Euler angle need to be defined and a rotation matrix $L_{B E}$ as equation (1) presented [8].

$$
L_{\mathrm{BE}}=\left(\begin{array}{lll}
\mathrm{c} \theta \mathrm{c} \psi & \mathrm{c} \theta \mathrm{s} \psi & -\mathrm{s} \theta \\
\mathrm{s} \phi \mathrm{s} \theta \mathrm{c} \psi-\mathrm{c} \phi \mathrm{s} \psi & \mathrm{s} \phi \mathrm{s} \theta \mathrm{s} \psi+\mathrm{c} \phi \mathrm{c} \psi & \mathrm{s} \phi \mathrm{c} \theta \\
\mathrm{c} \phi \mathrm{s} \theta \mathrm{c} \psi+\mathrm{s} \phi \mathrm{s} \psi & \mathrm{c} \phi \mathrm{s} \theta \mathrm{s} \psi-\mathrm{s} \phi \mathrm{c} \psi & \mathrm{c} \phi \mathrm{c} \theta
\end{array}\right)
$$

where $\theta, \phi$ and $\varphi$ are yaw, roll and pitch angle, respectively.

There are three phases of the tilt rotor during its flight phase.

1) Helicopter mode. In the Fig.1, the main features of this aircraft is a nacelle is located at the position which far from the wing root. When the angle between the nacelle and the body is 90 , this aircraft is a helicopter rotor.

2) Fixed wing mode. The aircraft is similar to a fixed wing aircraft with four propellers when the rotor nacelle parallel with the body.

3) Transition mode. The process of flight between the helicopter mode and the fixed wing mode is called a transition mode. 


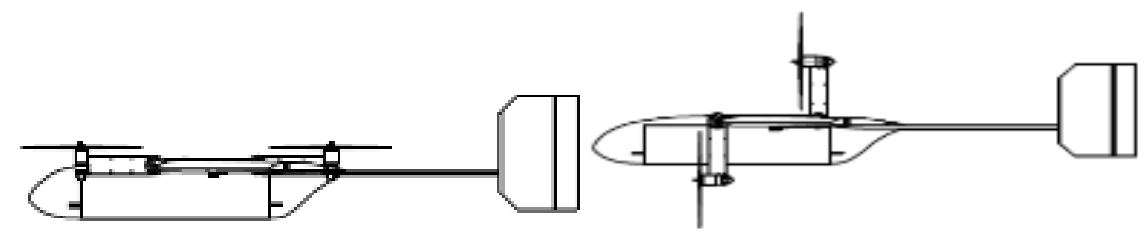

Fig. 1 Tilt Rotor Aircraft

Kinematics model. Using the equation (1) gives the aircraft translational kinematics equation:

$$
\left(\begin{array}{c}
\dot{x} \\
\dot{y} \\
\dot{z}
\end{array}\right)=L_{B E}^{T}\left(\begin{array}{l}
u \\
v \\
w
\end{array}\right)
$$

where $u, v$ and $w$ are the velocity of aircraft in the inertial frame. And the aircraft rotating kinematics equation can be given as follows:

$$
\left(\begin{array}{l}
\dot{\phi} \\
\dot{\theta} \\
\dot{\psi}
\end{array}\right)=\left(\begin{array}{ccc}
1 & \sin \phi \tan \theta & \cos \phi \tan \theta \\
0 & \cos \phi & -\sin \phi \\
0 & \sin \phi \sec \theta & \cos \phi \sec \theta
\end{array}\right)\left(\begin{array}{l}
p \\
q \\
r
\end{array}\right)
$$

where $p, q$ and $r$ are angular position states in the body frame.

Dynamic model. The aircraft translational and rotating dynamic equations are as the equation (4) and (5):

$$
\left(\begin{array}{c}
\dot{u} \\
\dot{v} \\
\dot{w}
\end{array}\right)=\left(\begin{array}{c}
r v-q w \\
p w-r u \\
q u-p v
\end{array}\right)+\frac{1}{m}\left(\begin{array}{c}
f_{x} \\
f_{y} \\
f_{z}
\end{array}\right)
$$

where $f_{x}, f_{y}$ and $f_{z}$ are the externally forces in the body frame.

$$
\left(\begin{array}{c}
\dot{p} \\
\dot{q} \\
\dot{r}
\end{array}\right)=\left(\begin{array}{c}
\Gamma_{1} p q-\Gamma_{2} q r+\Gamma_{3} l+\Gamma_{4} n \\
\Gamma_{5} p r-\Gamma_{6}\left(p^{2}-r^{2}\right)+\frac{1}{J_{y}} m \\
\Gamma_{7} p q-\Gamma_{1} q r+\Gamma_{4} l+\Gamma_{8} n
\end{array}\right)
$$

where,

$$
\begin{aligned}
& \Gamma_{1}=\frac{J_{x z}\left(J_{x}-J_{y}+J_{z}\right)}{\Gamma}, \quad \Gamma_{2}=\frac{J_{z}\left(J_{z}-J_{y}\right)+J_{x z}^{2}}{\Gamma}, \quad \Gamma_{3}=\frac{J_{z}}{\Gamma}, \quad \Gamma_{4}=\frac{J_{x z}}{\Gamma} \\
& \Gamma_{5}=\frac{J_{z}-J_{x}}{J_{y}}, \quad \Gamma_{6}=\frac{J_{x z}}{J_{y}}, \quad \Gamma_{7}=\frac{J_{x}\left(J_{x}-J_{y}\right)+J_{x z}^{2}}{\Gamma}, \quad \Gamma_{8}=\frac{J_{x}}{\Gamma}
\end{aligned}
$$

$J_{i j}(i, j=x, y)$ are elements of the inertial moment.

\section{Force and moment of transition mode}

Equation (7) and (8) are the pressure before and after air through the blade motor, with the application of Bernoulli equation, respectively.

$$
\begin{gathered}
P_{\text {up }}=P_{0}+\frac{1}{2} \rho V_{a}^{2} \\
P_{\text {down }}=P_{0}+\frac{1}{2} \rho V_{\text {exit }}^{2}
\end{gathered}
$$

where, $P_{\text {up }}, P_{\text {down }}$ are the pressures of air before and after it flowing through the rotors, 
respectively. $P_{0}$ is the hydrostatic pressure. $V_{\text {exit }}$ is the velocity of the air flowing through the rotors. Ideally, there is an approximate equation (9) without considering the transfer of rotors.

$$
V_{\text {exit }}=k_{\text {motor }} \delta_{t}
$$

where, $k_{\text {motor }}$ is parameter of rotor, $\delta_{t}$ is the control variable of throttle. The thrust of rotors can be given as the equation (10) :

$$
F_{p}=S_{\text {prop }} C_{\text {prop }}\left(P_{\text {up }}-P_{\text {down }}\right)=\frac{1}{2} \rho S_{\text {prop }} C_{\text {prop }}\left[\left(k_{\text {motor }} \delta_{t}\right)^{2}-V_{a}^{2}\right]
$$

where, $S_{\text {prop }}$ is the area of paddle flows, $C_{\text {prop }}$ is the non-dimensional parameter of thrust.

Therefore, the sum of the thrust of four rotors is shown as equation (11):

$$
\mathbf{f}_{p}=4 \times \frac{1}{2} \rho S_{\text {prop }} C_{\text {prop }}\left(\begin{array}{c}
{\left[\left(k_{\text {motor }} \delta_{t}\right)^{2}-V_{a}^{2}\right] \cos \tau} \\
0 \\
{\left[\left(k_{\text {motor }} \delta_{t}\right)^{2}-V_{a}^{2}\right] \sin \tau}
\end{array}\right)
$$

where, $\tau$ is the tilting angle of rotor relative to the symmetry plane.

It is necessary to guarantee the controllability of the aircraft in the transition mode. So the rotation rate of the rotors needs to be equal and the rotation direction is opposite. Therefore, the moment of rotors is zero, and the moment of transition mode as follows:

$$
\mathbf{m}_{p}=\left(\begin{array}{c}
M_{1}-M_{2}+M_{3}-M_{4} \\
0 \\
0
\end{array}\right)=\left(\begin{array}{l}
0 \\
0 \\
0
\end{array}\right)
$$

\section{Simulation and Experimental Verification}

Tilt rotor aircraft can improve flight performance to a great extent, however, it also brings many new problems and technical difficulties. The control method of the tilt rotor aircraft is complex, especially in the transition mode, because it is necessary to control the rudder and rotor at the same time. The difficulty of the transition mode is the stability of the aircraft when the rotor position changes. Therefore, the primary task of the transition mode is to guarantee the stability of it. And a simulation model is established as the Fig.3 shows.

Because of the nonlinear of model, S-function is introduced to solve this problem. S-function which is the abbreviation of system function has a special format to help users to make Simulink modules by themselves [9-11]. It can avoid using a lot of Simulink module and maintain part of the nonlinear characteristics of the model. As shown in Fig.2, the Dynamic module is S-function. The function consists of equation (2)-(5), which are the differential equations of state of the aircraft.

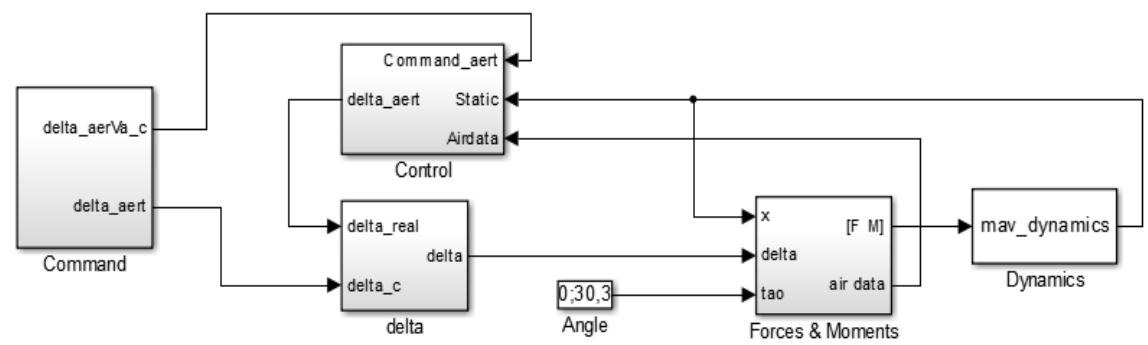

Fig.2 Simulation Model

The main function of Forces \& Moment module, which use a multiple input and output function, is to calculate the force and moment of the aircraft. With the deflection of control surfaces, feedback of state variables and changes of rotor position input, the force, moment, wind speed and attack angle are outputted.The Control module is encapsulated in PID controller. Use the following control rate: 


$$
\begin{aligned}
& \Delta \delta_{a}=k_{p, \phi}\left(\phi^{d}-\phi\right)+k_{i, \phi} \int\left(\phi^{d}-\phi\right) d t+k_{d, \phi} \frac{d\left(\phi^{d}-\phi\right)}{d t} \\
& \Delta \delta_{e}=k_{p, \theta}\left(\theta^{d}-\theta\right)+k_{i, \theta} \int\left(\theta^{d}-\theta\right) d t+k_{d, \theta} \frac{d\left(\theta^{d}-\theta\right)}{d t} \\
& \Delta \delta_{r}=k_{p, \psi}\left(\psi^{d}-\psi\right)+k_{i, \psi} \int\left(\psi^{d}-\psi\right) d t+k_{d, \psi} \frac{d\left(\psi^{d}-\psi\right)}{d t} \\
& \Delta \delta_{t}=k_{p, V_{a}}\left(V_{a}^{d}-V_{a}\right)+k_{i, V_{a}} \int\left(V_{a}^{d}-V_{a}\right) d t+k_{d, V_{a}} \frac{d\left(V_{a}^{d}-V_{a}\right)}{d t}
\end{aligned}
$$

The delta module is the relationship between the expected parameter and feedback parameter.

$$
\begin{aligned}
& \delta_{a}=\delta_{a}^{d}+\Delta \delta_{a} \\
& \delta_{e}=\delta_{e}^{d}-\Delta \delta_{e} \\
& \delta_{r}=\delta_{r}^{d}-\Delta \delta_{r} \\
& \delta_{t}=\delta_{t}^{d}+\Delta \delta_{t}
\end{aligned}
$$

The Angle module is signal of rotor position rotate and a pulse function in time which is shown as Fig.3.

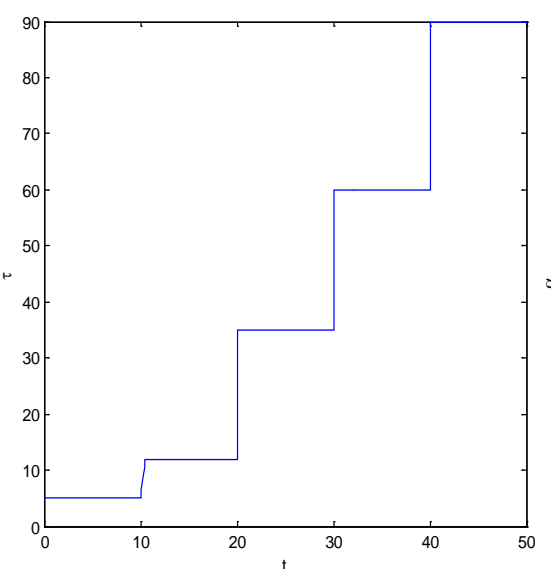

Fig.3 Pulse of Rotor Angle Change

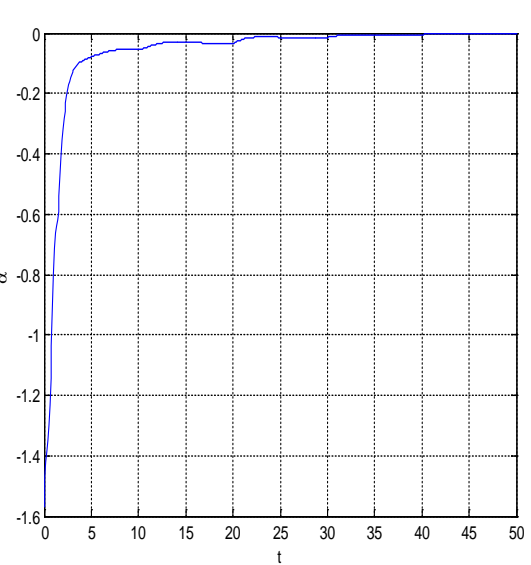

Fig.4 Change of Attack Angle

In Fig.4, the value of pulse is larger and larger as time increasing. When the rotor angle began to change, the speed of vehicle is small and the aerodynamic has little effect on it. At this moment, rotors need to support lift to the aircraft, so the change of rotor angle need to be small every time. At the end of the transition mode, the flying speed is relatively large and the weight of lift provided from rotors is small. The change of the rotor angle can be larger than it was in order to end the transition mode quickly.

Taking a type of tilt rotor aircraft as example to model and calculate. The main parameters are shown in the table1.

Table1 Main Parameters

\begin{tabular}{cccccc}
\hline Mass & Span & Wing area & Paddle length & Initial velocity & Initial Elevating angle \\
\hline $13.5 \mathrm{~kg}$ & $2.06 \mathrm{~m}$ & $0.42 \mathrm{~m}^{2}$ & $0.5 \mathrm{~m}$ & $1 \mathrm{~m} / \mathrm{s}$ & $0.2 \mathrm{rad}$ \\
\hline
\end{tabular}

Fig.4-Fig.8 are results of simulation.Fig.4 shows the change of attack angle. At the beginning of transition mode, the aircraft tends to horizon, while the velocity is vertical which leads to an almost 90 degree attack angle. As time increasing, the $\mathrm{X}$ axis of the body frame tends to horizon too, so the attack angle changes to zero gradually.

Fig. 5 shows the change of flight height. From the figure, the slope of height curve is large at the initial moment because of lift provided by rotors. With the decrease of lift in vertical direction, the flight height increases more and more gently and achieves at $55.7 \mathrm{~m}$ finally. 


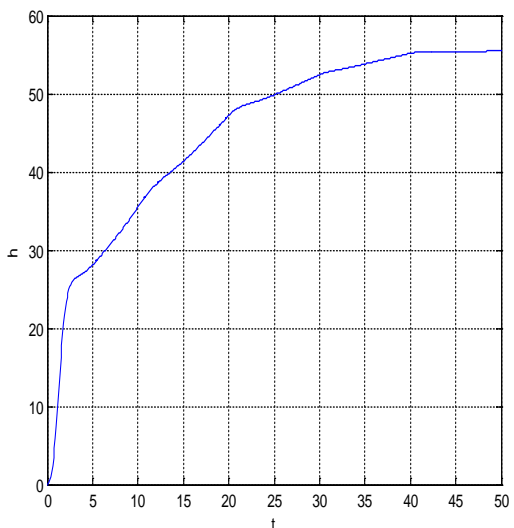

Fig.5 Change of Flight Height

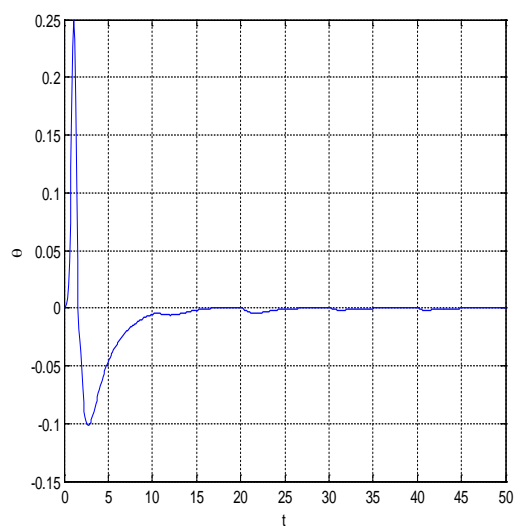

Fig.6 Change of Pitch Angle

As shown in Fig.6, the pitch angle of aircraft changes greatly in the beginning. The aircraft has a horizontal speed which produces pitching moment on the center of the aircraft at that time, although the aerodynamic lift is relatively smaller. Then the moment introduces a positive pitch angle. The signal of pitch angle flows to the PID controller as feedback which causes the elevator changes.

Fig.7 shows the elevator angle changes. With the adjustment of PID controller, elevator began to balance the pitching moment at the same time. With the pitch angle changing, they all tend to zero finally.

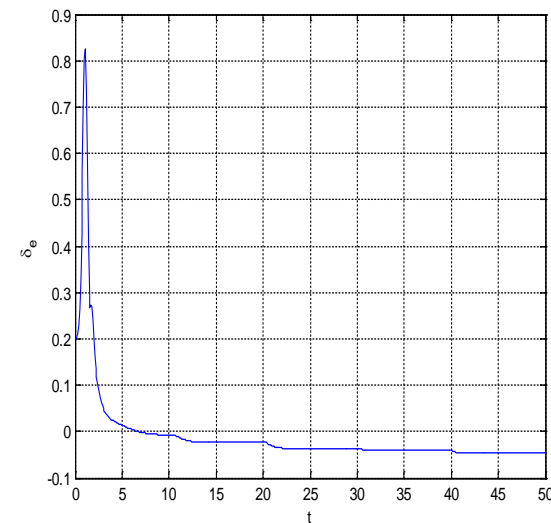

Fig.7 Change of Elevator Angle

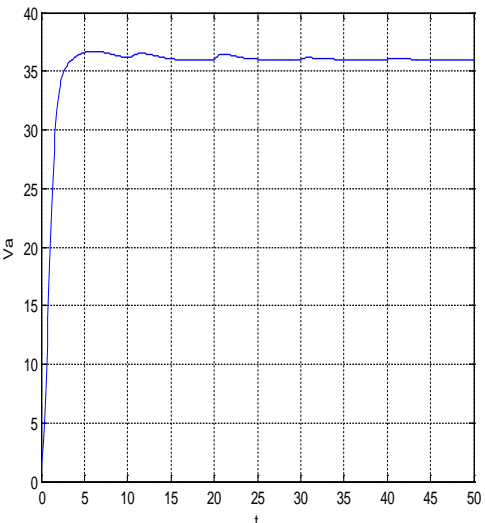

Fig.8 Change of Velocity

Fig.8 shows the change of vehicle speed. The velocity is gradually increasing along with the simulation time and floating up and down at the given velocity.

\section{Conclusion}

This paper establishes a six degrees of freedom dynamic model of tilt rotor aircraft, and a PID controller is adopted to control the model. S-function is used to handle the dynamic equation nonlinearity. Finally, a dynamic simulation model of a certain aircraft in the transition mode is established in Simulink. From the results of simulation, the following conclusions can be obtained:

1) In the transition mode, the attitude of aircraft changes in allowable range and satisfied the motion laws. The dynamic model can be applied to a general tilt rotor aircraft.

2) The S-function can solve the nonlinearity of dynamic model better and retain nonlinearity in other parts of the model, which makes the simulation results more accurate.

Altogether, the dynamic model of tilt rotor aircraft in transition mode based on S-function established in this paper can provide a reference for analysis of tilt rotor aircraft dynamic and design of flight control system. 


\section{Reference}

[1] Song Yanguo, Wang Huanjin. Design of Flight Control System for a Small Unmanned Tilt Rotor Aircraft: submitted to Chinese Journal of Aeronautics(22)2009:250-256.

[2] Li Haixu, Qu Xiangju and Wang Weijun. Multi-body Motion Modeling and Simulation for Tilt Rotor Aircraft: submitted to Chinese Journal of Aeronautics (23) 2010 :415-422.

[3] SHA Hong-wei. Research on flight dynamics of tilt rotor aircraft base on Simulink : submitted to Journal of Flight Dynamics(5)2012:389-397.

[4] YANG Xili , ZHU Jihong and HUANG Xingli etc. Modeling and Simulation for Tiltrotor Airplane: submitted to Acta Aeronau Tica Et Astronau Tica Sinica(4)2006:584-587

[5] Zhu Y. A mathematical model of tilt-rotor flight dynamics and investigation of flight control in transition mode PhD thesis, Nanjing: Nanjing University of Aeronautics and Astronautics, 2005.

[6] Jinoh Lee, Changsun Yoo and Yea-Seok Park. An experimental study on time delay control of actuation system of tilt rotor unmanned aerial vehicle: submitted to Mechatronics (22,2)2012:184-194

[7] WU Ri shan, YANG Jiajun, JIN Guozhen. The Study on the Dynamics Simulation of Mechanism Based on Simulink S-function: submitted to Journal of Hubei Polytechnic University(3)55-57.

[8]Randal W.Beard, Timothy W.Mclain "Small Unmanned Aircraft” Princeton University Press.2011

[9] Zhiyong Zhang “Be good at Matlab R2011a” Beihang university press,2011.

[10] Shen J W, Floros M, Lee M K, et al. Multi-body dynamicssimulation of a tilt rotor UAV. The 2nd International Basic Research Conference on Rotorcraft Technology. 2005.

[11] Heron P J, Kunz D, Nikravesh P, et al. A multibodyapproach to modeling tilt-wing rotorcraft dynamics: submitted to AlAA-1992-0487, 1992. 